\title{
Comparison of Flair MRI to Contrast Enhanced T1W MRI in Selected Intracranial Pathologies for a Patient Population in North Kerala, India
}

\author{
Aarif Latheef ${ }^{1}$, Saanida M. P. ${ }^{2}$, Juvaina P. ${ }^{3}$, Naufal P4 \\ ${ }^{1}$ Department of Radiodiagnosis, Government Medical College, Kozhikode, Kerala, India. \\ ${ }^{2}$ Department of Radiodiagnosis, Government Medical College, Kozhikode, Kerala, India. \\ ${ }^{3}$ Department of Radiodiagnosis, Government Medical College, Kozhikode, Kerala, India. \\ ${ }^{4}$ Department of Radiodiagnosis, Government Medical College, Kozhikode, Kerala, India.
}

\section{ABSTRACT}

\section{BACKGROUND}

Fluid Attenuated Inversion Recovery (FLAIR) MRI of brain is sensitive in detecting parenchymal lesions, extra axial lesions such as meningoencephalitis, and leptomeningeal tumoural disease. We wanted to compare gadolinium enhanced T2W FLAIR with PC-T1W (post contrast T1 weighted) MRI sequence in detection and evaluation of meningeal and parenchymal lesions. We also wanted to compare gadolinium enhanced T1W FLAIR with PC-T1W MRI sequence in detection and evaluation of meningeal and parenchymal lesions.

\section{METHODS}

65 patients with clinical diagnosis of cerebral meningitis or intracranial tumoural disease were scanned on $1.5 \mathrm{~T} 16$ channel MRI scanner. A standard brain protocol was used, followed by PC-T1W and post contrast FLAIR, with PC-T1W MR preceding post contrast FLAIR. Images were assessed independently by 2 radiologists who marked the technique they thought had made the meningeal and parenchymal disease more conspicuous. In case of disagreement the radiologists made a consensus determination.

\section{RESULTS}

PC-T2 FLAIR showed better enhancement in $100 \%$ of the intra axial lesions compared to PC-T1W images. However, PC-T1 FLAIR showed better enhancement than PC-T1W images in only $81 \%$ of the same. PC-T2 FLAIR showed better enhancement in $100 \%$ of the extra-axial lesions compared to PC-T1W images. However, PC-T1 FLAIR showed less enhancement in all the extra axial lesions.

\section{CONCLUSIONS}

Contrast enhanced T2 FLAIR MRI showed better enhancement of parenchymal and meningeal lesions. PC-T2 FLAIR detected more number of lesions when compared to PC-T1W, aided better delineation of extra-axial mass lesions due to superior enhancement, and had a better demarcated dural tail. Post-contrast T2 FLAIR images should be included in routine protocol for contrast MRI brain study.

\section{KEY WORDS}

FLAIR (Fluid Attenuated Inversion Recovery), PC-T1W Weighted Image, Extra Axial, Intra Axial Lesions
Corresponding Author:

Saanida M. P.,

Assistant Professor,

Department of Radiodiagnosis,

Government Medical College,

Kozhikode-673006, Kerala, India.

E-mail: saanida.monthampally@gmail.com

DOI: $10.14260 /$ jemds/2020/188

Financial or Other Competing Interests: None.

How to Cite This Article:

Latheef A, Saanida MP, Juvaina $P$, et al. Comparison of flair MRI to contrast enhanced T1W MRI in selected intracranial pathologies for a patient population in North Kerala, India. J. Evolution Med. Dent. Sci. 2020;9(11):875878, DOI: 10.14260/jemds/2020/188

Submission 25-01-2020,

Peer Review 24-02-2020

Acceptance 26-02-2020,

Published 16-03-2020.

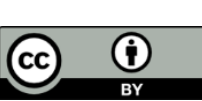




\section{BACKGROUND}

It is fairly well known in the literature e.g.[1],[2],[3] that Fluid Attenuated Inversion Recovery (FLAIR) MRI of brain is sensitive in detecting parenchymal lesions, extra axial lesions such as meningoencephalitis, and leptomeningeal tumoural disease. Our study establishes relevance of this finding to a population of North Kerala, with aim to propose inclusion of Post-Contrast FLAIR sequence in protocol of the centre. Our work specifically aims-

- To compare Post-Contrast T2-weighted FLAIR (called T2WF) with PC-T1W MRI sequence in detection and evaluation of meningeal lesions.

- $\quad$ To compare T2WF with PC-T1W MRI imaging sequence in detection and evaluation of parenchymal lesions.

- $\quad$ To compare T1WF with PC-T1W MR imaging sequence in detection and evaluation of parenchymal disease.

FLAIR imaging is superior in the diagnosis of leptomeningeal infections and metastatic lesions because the suppression of CSF signal causes better delineation of meningeal lesions. However, FLAIR is not part of protocol for such cases in centres such as our institution Govt. Medical College, Kozhikode, Kerala, India - mostly owing to paucity of available scanner time. Our study establishes that T2F is better in detection of meningeal enhancement.

Such studies are relevant because contrast enhanced MRI aids in early diagnosis of meningeal infection and metastatic lesions even when CSF culture reports are falsely negative. Thus, good diagnostic ability would help in preventing complications like brain abscess and infarction. The superiority of FLAIR imaging in the diagnosis of meningeal lesions may also be due to fact that FLAIR sequences are more sensitive than T1W imaging for demonstrating low concentrations of gadolinium. Unlike T1W images contrast enhanced FLAIR images do not show enhancement in the vessels with slow flow of blood. This will help in better detection of meningeal enhancement on post contrast T2 FLAIR images. The effectiveness of FLAIR imaging in detection of leptomeningeal diseases may also be due to the additive T1 and T2 effect of FLAIR. In acute pathologies, rather than chronic ones, a small amount of gadolinium may not shorten the T1 time of CSF enough to be picked up by a PC-T1W scan, hence $\mathrm{T} 2 \mathrm{~F}$ is likely to perform better. In chronic infections there is thickening of the meninges with increased vascularity which in turn will lead to retention of more contrast causing shortening of $\mathrm{T} 1$ time causing enhancement. In both types of FLAIR: T1 and T2 effects of various tissues supplement each other adding to signal intensity. T2F showed distinctly lesion enhancement and perilesional oedema on the same image which helps in tumour quantification and follow up.

In addition, note that Meningitis is an important lifethreatening emergency requiring prompt diagnosis and treatment. Any delay in diagnosis and antibiotic administration will lead to adverse prognosis in case of bacterial meningitis. Infective meningitis including tuberculous and bacterial meningitis is the leading cause of stroke in young patients. Bacterial meningitis is the leading cause of morbidity in children below the age of 5 years. Diagnosis of meningitis is established by history, clinical examination and laboratory evaluation but the ability to detect and differentiate intracranial infection has markedly improved with introduction of contrast enhanced MRI. For a long time, PC-T1W images are the preferred sequence for detection of meningitis, our study aims to establish utility of $\mathrm{T} 2 \mathrm{~F}$ in such circumstances.

\section{METHODS}

This is a descriptive study, conducted among 65 patients diagnosed of cerebral meningitis or intracranial tumoural disease referred to Department of Radiodiagnosis, Government Medical College, Kozhikode, (also known as Calicut), Kerala, India, over a period of 18 months from Jan 2017 to Jun 2018.

\section{Inclusion Criteria}

- Patients with clinical diagnosis of cerebral meningitis or intracranial tumoural disease referred to Department of Radio diagnosis, Government Medical College Kozhikode, Kerala, India.

- Patients who are willing to participate in the study.

\section{Exclusion Criteria}

- Patients not consenting to be part of the study.

- Patients who have contraindications for MRI.

- Patient who have contraindications for Gd contrast.

\section{Sample Size}

Kremer et al.[1] recommends a sample size of 36 for a similar diagnostic setting. We reproduce the sample size calculation: using the formula (4pq)/d ${ }^{2}$ of which ' $\mathrm{p}$ ' is the sensitivity of FLAIR (taken as 90), ' $q$ ' has the formula (100-p) (equals 10) and ' $d$ ' is the allowable error in percentage (taken as 10). Thus, sample size arrived at is 36 , cases available are 65 in number.

MR images were assessed independently for the presence or absence of leptomeningeal, patchy meningeal or parenchymal signal abnormality or contrast enhancement and opinion was sought as to which technique made the meningeal and parenchymal disease more conspicuous. In case of disagreement the radiologist made consensus determination.

\section{RESULTS}

PC-T1W MR images, T2F and T1F images were assessed by experienced radiologists for the conspicuity, delineation and number of the lesions. This study included 65 patients with mean age of $40.7 \mathrm{yrs}$. with standard deviation of 22 . Out of the 65 patients studied 32 were male (49.2\%) and 33 were female (50.8\%). Comparative results are provided in the form of tables. Comparative result of enhancement in intra axial parenchymal lesions in PC-T1W, T2F and T1F are presented next as Table 2 .

\begin{tabular}{|ccc|}
\hline Location of Intra-Cranial Lesion & Frequency & Percentage \\
Intra axial & 38 & 58.46 \\
Extra axial & 27 & 41.54 \\
Total & $\mathbf{6 5}$ & $\mathbf{1 0 0}$ \\
\hline Table 1. Frequency of Lesions in Patients \\
\hline
\end{tabular}




\begin{tabular}{|ccc|}
\hline Lesions & Frequency & Percentage \\
Tuberculoma & 3 & 7.89 \\
High grade glioma & 12 & 31.57 \\
Low grade glioma & 5 & 13.15 \\
Medulloblastoma & 2 & 5.26 \\
Hemangioblastoma & 1 & 2.63 \\
Tumefactive demyelination & 1 & 2.63 \\
Subependymoma & 1 & 2.63 \\
Ependymoma & 1 & 2.63 \\
Metastasis & 11 & 28.94 \\
Non-Hodgkin's lymphoma & 1 & 2.63 \\
Total & $\mathbf{1 0 0}$ \\
\hline \multicolumn{2}{c}{ Table 2. Intra-Axial Lesions in Patients } \\
\hline
\end{tabular}

\section{Detection of Intra-Axial Parenchymal Lesions by T2F}

This study included 38 intra axial lesions. T2F showed better enhancement in all the parenchymal lesion studied as compared to PC-T1W images $(100 \%)$. T2F showed better delineation of enhancing lesion from the non-enhancing perilesional FLAIR hyperintense oedema, this in turn led to better delineation of tumour and further to better follow up of the lesion in the course of treatment. In patients with multiple lesions, T2F images showed more number of lesions in 3 cases and equal number of lesions in rest of the cases as compared to PC-T1W. T2F helped in delineation of wall characteristics in all the cases of tuberculomas included in the study. In all the 11 cases of parenchymal metastatic lesions T2F showed brighter enhancement as compared to PC-T1W images. Of the 11 parenchymal metastatic lesions studied T2F show better enhancement than PC-T1W images.

\section{Detection of Intra-Axial Parenchymal Lesions by T1F Images}

Out of the 38 cases of intra axial parenchymal lesions included in this study, only in 31 cases did T1F images show better enhancement compared to PC-T1W images (81.5\%). However, in patients with multiple lesions, in 1 case T1F showed more number of lesions than PC-T1W. In rest of the cases PC-T1W FLAIR and PC-T1W showed same number of lesions. In all the 5 cases of low-grade glioma the studied T1F images showed less enhancement compared to PC-T1W. Of the 11 parenchymal metastatic lesions studied T1F showed better enhancement than PC-T1W images.

\begin{tabular}{|ccc|}
\hline Enhancement & Frequency & Percentage \\
More & 31 & 81.57 \\
Less & 6 & 15.78 \\
Same & 1 & 2.64 \\
Total & $\mathbf{3 8}$ & $\mathbf{1 0 0}$ \\
\hline Table 3. Comparison of T1F with PC-T1W in Intra Axial Lesions \\
\hline
\end{tabular}

\begin{tabular}{|ccc|}
\hline Lesions & Frequency & Percentage \\
& 4 & 14.81 \\
Tubercular meningitis & 7 & 27.92 \\
Fungal meningitis & 2 & 7.40 \\
Toxoplasmosis & 1 & 3.7 \\
Viral meningitis & 2 & 7.40 \\
Acoustic schwannoma & 2 & 7.40 \\
Meningioma & 8 & 29.62 \\
Epidermoid cyst & 1 & 3.7 \\
Total & $\mathbf{2 7}$ & $\mathbf{1 0 0}$ \\
\hline Table 4. Distribution of Extra Axial Lesion in the Study Population \\
\hline
\end{tabular}

\section{Flair in Infectious Meningeal Pathology}

16 cases with clinical diagnosis of leptomeningitis where studied. Of these, in 15 cases meningeal enhancement was easily identified in T2F images when compared to PC-T1W images (93.75\%). However, PC-T1W images rendered difficult the differentiation of meningeal enhancement from vascular enhancement. CSF studies were positive in all 16 cases. T1F images demonstrated less enhancement to PC-T1W in 15 cases (93.75\%). All the 16 cases of leptomeningeal enhancement were followed up and clinical diagnosis was based on patient symptoms, CSF analysis and therapeutic response (R). Comparative result of meningeal enhancement on PC-T1W and T2F is presented as Table 5.

\section{Flair in Detection of Extra axial Lesions}

Of the 11 extra axial lesions included in our study, T2F showed better enhancement in all the cases when compared to PCT1W images $(100 \%)$. In all these extra axial lesions studied T1F showed less enhancement than PC-T1W. Our study included 8 cases of meningioma. In all the cases of meningioma studied, the dural tail was better in T2F images when compared to PC-T1W images (100\%). Out of the 8 cases of meningioma $\mathrm{T} 1 \mathrm{~F}$ showed less enhancement of dural tail in 6 cases when compared to PC-T1W images (75\%). In the other 2 cases of meningioma studied $\mathrm{T} 1 \mathrm{~F}$ images showed similar enhancement of dural tail when compared to PC-T1W images (25\%). In all the 11 extra axial lesions studied CSF cleft was seen. T2F imaging showed better delineation of the CSF cleft when compared to PC-T1W (100\%). In all these cases the delineation of the CSF cleft was similar in both PC-T1W images and $\mathrm{T} 1 \mathrm{~F}$ images (100\%).

\begin{tabular}{|ccc|}
\hline Enhancement & Frequency & Percentage \\
More & 15 & 93.75 \\
Less & 1 & 6.25 \\
Same & 0 & 0 \\
Total & $\mathbf{1 6}$ & $\mathbf{1 0 0}$ \\
\hline Table 5. Comparison of T2F with PCT1 in Meningitis \\
\hline
\end{tabular}

\section{DISCUSSION}

We have obtained results similar to Kremer et al.[1] which compares unenhanced, enhanced, delayed enhanced FLAIR images with enhanced T1W images in a smaller number of 10 patients and concluded that delayed enhanced FLAIR improves the detection of leptomeningeal infections and tumoural disease when compared to PC-T1W. Note also that Mathews et al.[3] reported that T2F is superior to PC-T1W images in detection of superficial abnormalities such as meningeal disease. We found that in T2F images most of the intra-axial parenchymal lesions appeared more conspicuous than in PC-T1W. Note however that this is not an overly established view. Mills et al.[4] in a review article on advanced MRI biomarkers of cerebral metastasis said that sequences like contrast enhanced multi shot ECHO planar FLAIR and pre and post contrast inversion recovery T1W sequences instead of conventional T1 spin ECHO sequences have no advantage in improving lesion conspicuity. In an invitro phantom study conducted by Mamourian et al.[5] FLAIR MRI was more sensitive to gadolinium effects at 4 times lower concentration than T1W MRI was.

In studies done by Singh et al[6] and Galassi et al[7] showed that contrast enhanced T1W image with fat suppression to be superior to contrast enhanced FLAIR imaging in leptomeningeal disease. In this study, both early and delayed post contrast FLAIR images were used alternatively. These studies also included chronic meningeal pathologies. In our study T2F was found to be useful in differentiating between metastatic disease and high-grade glioma because of greater degree of signal changes representing oedema in metastatic 
disease. In our study $\mathrm{T} 1 \mathrm{~F}$ was not sensitive in detection of leptomeningeal enhancement. Leaking of gadolinium into CSF may shorten the T1 of CSF so that longitudinal magnetisation recovers before the null point of CSF on T2 FLAIR images. This leakage of gadolinium is although not enough for the recovery of CSF signal intensity on T1 FLAIR images because of the shorter TI time. However, T1F provided better anatomic delineation with better enhancement of high-grade tumours, infectious ring enhancing lesions and large metastatic tumours.

In a descriptive analytical study conducted by Shobiri et al.[2] on 49 patients with leptomeningeal diseases the degree of post contrast enhancement was compared. The study concluded that post contrast FLAIR image are superior in detecting meningeal lesions and can be used as an adjunct to PCT1W for detection of leptomeningeal lesions. A study conducted on 9 patients with clinical diagnosis of meningeal carcinomatosis (Tsuchiya et al.[8]) compared T2F and plain FLAIR images to other sequences and concluded that plain T2F and contrast enhanced FLAIR images were superior for evaluating meningeal carcinomatosis than T2 weighted images or even PC-T1W. Similarly, pointing to a contrary direction, a study done on 68 patients with cytologically proven leptomeningeal metastasis, Singh et al,[6] concluded that T2F is less sensitive than PC-T1W MR sequences.

Jeevanandham ${ }^{[9]}$ in a study on 27 patients with meningitis compared 3D T2F, T1 SPACE, and MP RAGE sequences to determine the best sequence for the detection of leptomeningeal abnormalities in a 3T MRI. Their study concluded that SPACE images were superior to even 3D T2 FLAIR and MP RAGE in detection of leptomeningeal diseases. Vaswani et al.[10] conducted a study to determine the diagnostic accuracy of T2F in diagnosing meningitis and found that $\mathrm{T} 2 \mathrm{~F}$ was $96 \%$ sensitive and $85.7 \%$ specific for diagnosing meningitis. PC-T1W was $68 \%$ sensitive and $85.71 \%$ specific for the same, indicating a specificity on par with T2F. The conclusion made in this study was that $\mathrm{T} 2 \mathrm{~F}$ is more sensitive albeit not specific-over PC-T1W in the diagnosis of meningitis.

\section{CONCLUSIONS}

In our study, $\mathrm{T} 2 \mathrm{~F}$ showed better enhancement of parenchymal and meningeal lesions. T2F detected more lesions than PC$\mathrm{T} 1 \mathrm{~W}$, also aiding in better delineation of extra-axial mass lesions and demonstrating superior enhancement with better demarcation of dural tail. Hence the recommendation that T2F images should be included in the routine protocol for contrast MRI brain study even in centres where there are time constraints do not favour the current scheme. T1F had no advantage compared to PC-T1W images in detection of meningeal lesions. T1F shows less enhancement when compared to PC-T1W images in extra-axial mass lesions. However, based on a restricted sample, it is also the case that
T1F showed better enhancement and delineation of high grade, vascular tumours and large tuberculomas compared to PC-T1W.

\section{Limitations}

This study was conducted with a relatively small sample size over a short period of time ( $90 \%$ confidence). Also, post contrast enhancement of intracranial lesions also depends on the delay in the scanning time. But due to the practical considerations, the timing varied from patient to patient.

\section{REFERENCES}

[1] Kremer S, Abu Eid M, Bierry G, et al. Accuracy of delayed post-contrast FLAIR MR imaging for the diagnosis of leptomeningeal infectious or tumoural diseases. J Neuroradiol 2006;33(5):285-91.

[2] Shobiri E, Ghadiri K, Tanghatari B, et al. Comparing FLAIR and contrast-enhanced T1-weighted MRI in meningeal diseases. J Kermanshah Univ of Med Sci 2013;17(5):30915.

[3] Mathews VP, Caldemeyer KS, Lowe MJ, et al. Brain: gadolinium-enhanced fast fluid-attenuated inversionrecovery MR imaging. Radiology 1999;211(1):257-63.

[4] Mills SJ, Thompson G, Jackson A. Advanced magnetic resonance imaging biomarkers of cerebral metastases. Cancer Imaging 2012;12(1):245-52.

[5] Mamourian AC, Hoopes PJ, Lewis LD. Visualization of intravenously administered contrast material in the CSF on fluid-attenuated inversion-recovery MR images: An in vitro and animal- model investigation. Am J Neuroradiol 2000;21(1):105-11.

[6] Singh SK, Leeds NE, Ginsberg LE. MR imaging of leptomeningeal metastases: comparison of three sequences. Am J Neuroradiol 2002;23(5):817-21.

[7] Galassi W, Phuttharak W, Hesselink JR, et al. Intracranial meningeal disease: Comparison of contrast-enhanced MR imaging with fluid-attenuated inversion recovery and fatsuppressed T1-weighted sequences. Am J Neuroradiol 2005;26(3):553-9.

[8] Tsuchiya K, Katase S, Yoshino A, et al. Flair MR imaging for diagnosing intracranial meningeal carcinomatosis. Am J Roentgenol 2001;176(6):1585-8.

[9] Jeevanandham B. To compare post contrast 3D T2 FLAIR, T1-SPACE and MP-RAGE sequences to select the ideal sequence for leptomeningeal abnormalitites at $3 \mathrm{~T}$ MRI. Poster at ECR 2015: p. 1-35.

[10] Vaswani AK, Nizamani WM, Ali $M$, et al. Diagnostic accuracy of contrast-enhanced FLAIR magnetic resonance imaging in diagnosis of meningitis correlated with CSF analysis. Radiology 2014;2014:578986. http://dx.doi.org/10.1155/2014/578986. 